\title{
Problems in inverse scattering of approximate reflection coefficient measurements
}

\author{
Eric A. Kincanon * \\ Gonzaga University, Spokane, WA USA \\ *Corresponding author E-mail:kincanon@gonzaga.edu
}

\begin{abstract}
Because of the nonlinear nature of the Gelfand-Levitan equation, it may be a concern that a small difference in the reflection coefficient could lead to large changes in the corresponding potential. This paper considers this and shows that this need not be a concern. Though assumptions are made about the associated spectral measure function, these are not restrictive.
\end{abstract}

Keywords: Ambiguities; Gelfand-Levitan; Inverse Scattering; Reflection Coefficient; Spectral Function.

\section{Introduction}

Inverse scattering on the line is a well-known method to determine the potential $\mathrm{V}(\mathrm{r})$ given the reflection coefficient $\mathrm{R}(\mathrm{k})$. This is done by using the Gelfand-Levitan equation [1-3]:

$$
\mathrm{K}(\mathrm{r}, \mathrm{s})+\mathrm{G}(\mathrm{r}, \mathrm{s})+\int_{-\infty}^{\mathrm{r}} \mathrm{K}(\mathrm{r}, \mathrm{t}) \mathrm{G}(\mathrm{t}, \mathrm{s}) \mathrm{dt}=0
$$

Where $G(r, s)$, the spectral measure function, is the Fourier transform of the reflection coefficient $\mathrm{R}(\mathrm{k})$ :

$G(r, s)=\frac{1}{2 \pi} \int_{-\infty}^{+\infty} R(k) e^{-i k(r+s)} d k$

And $\mathrm{K}(\mathrm{r}, \mathrm{s})$ is related to the potential by:

$V(r)=-2 \frac{d K(r, r)}{d r}$

The typical procedure is to find $\mathrm{V}(\mathrm{r})$ by taking the Fourier transform of $R(k)$ to get $G(r, s)$ and then solve (1) for $K(r, s)$. Then (3) can be used to find the potential. The challenge arises in solving (1). This is typically done by successive iteration [4].

A potential concern in using this in practice is that $\mathrm{R}(\mathrm{k})$ cannot be measured perfectly. This paper considers how slightly different spectral measure functions, associated with slightly different $\mathrm{R}(\mathrm{k})$ affect the resulting calculated potential. This is done by looking at the expansion of two general $\mathrm{G}(\mathrm{r}, \mathrm{s})$ functions and assuming that the first $\mathrm{N}$ terms agree well. The corresponding $\mathrm{K}(\mathrm{r}, \mathrm{s})$ for each of these is found and the difference in the corresponding potentials is approximated.

\section{Calculations}

Solving (1) for $K(r, s)$ gives:

$$
K(r, s)=-G(r, s)-\int_{-\infty}^{r} K(r, t) G(t, s) d t
$$

Iterating this equation yields:

$$
\begin{aligned}
& K(r, s)= \\
& -G(r, s)+ \\
& \int_{-\infty}^{r} G(r, t) G(t, s) d t-\int_{-\infty}^{r} G(r, t) \int_{-\infty}^{r} G(t, u) G(u, s) d u+ \\
& \text { continued terms of iteration }
\end{aligned}
$$

Now consider two spectral measure functions that would be generated by (2) from slightly different $\mathrm{R}(\mathrm{k})$. (Expanding these functions in terms of exponentials is justified based in the usefulness of the exponential as the related to complete sets [5] and on known method for solving the Gelfand-Levitan equation. [6])

$$
\mathrm{G}_{1}(\mathrm{r}, \mathrm{s})=\sum_{\mathrm{n}=0}^{\infty} \mathrm{a}_{\mathrm{n}} \mathrm{e}^{\mathrm{n}(\mathrm{r}+\mathrm{s})}
$$

And

$\mathrm{G}_{2}(\mathrm{r}, \mathrm{s})=\sum_{\mathrm{n}=0}^{\infty} \mathrm{b}_{\mathrm{n}} \mathrm{e}^{\mathrm{n}(\mathrm{r}+\mathrm{s})}$

Since it assumed that these spectral measure functions have been generated by similar $\mathrm{R}(\mathrm{k})$ and that the series (6) and (7) converge, it is reasonable that the series agree up to some term of the sum $\mathrm{N}$. This means that to a good approximation it can be assumed that:

$\mathrm{G}_{1}(\mathrm{r}, \mathrm{s})=\mathrm{G}_{2}(\mathrm{r}, \mathrm{s})+\epsilon \mathrm{e}^{\mathrm{N}(\mathrm{r}+\mathrm{s})}$

Here $\varepsilon$ is taken as small. This allows for the $\mathrm{K}(\mathrm{r}, \mathrm{s})$ associated with (6) and (7) to be written in terms of each other. To do this consider the form of (5) associated with (6):

$$
\begin{aligned}
& \mathrm{K}_{1}(\mathrm{r}, \mathrm{s})= \\
& -\mathrm{G}_{1}(\mathrm{r}, \mathrm{s})+ \\
& \int_{-\infty}^{\mathrm{r}} \mathrm{G}_{1}(\mathrm{r}, \mathrm{t}) \mathrm{G}_{1}(\mathrm{t}, \mathrm{s}) d \mathrm{t}-\int_{-\infty}^{\mathrm{r}} \mathrm{G}_{1}(\mathrm{r}, \mathrm{t}) \int_{-\infty}^{\mathrm{r}} \mathrm{G}_{1}(\mathrm{t}, \mathrm{u}) \mathrm{G}_{1}(\mathrm{u}, \mathrm{s}) \mathrm{du}+ \\
& \text { continued terms of iteration }
\end{aligned}
$$

Now applying (8) to this equation yields: 


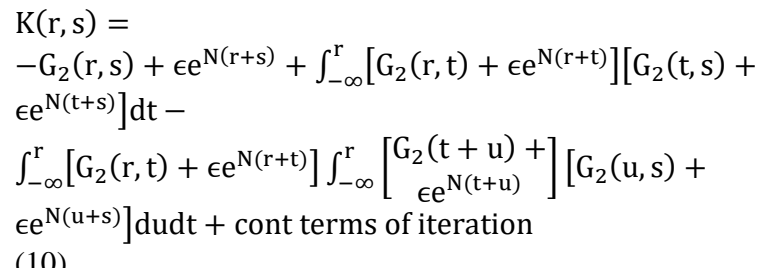

Getting rid of the brackets, keeping only terms that are linear in and grouping terms so that correspond to $K_{2}(r, s)$, leaves:

$K_{1}(r, s)=K_{2}(r, s)+\epsilon e^{N(r+s)}+\epsilon e^{N r} \int_{-\infty}^{r} G_{2}(t, s) d t+$
$\epsilon e^{N s} \int_{-\infty}^{r} G_{2}(r, t) d t$

What is of concern here is whether the different spectral measure functions correspond to significantly different potentials as given by (3). The size of this difference can be seen by examining the difference in the two $\mathrm{K}(\mathrm{r}, \mathrm{s})$ terms. So, rewriting (11) yields:

$\mathrm{K}_{1}(\mathrm{r}, \mathrm{s})-\mathrm{K}_{2}(\mathrm{r}, \mathrm{s})=\epsilon \mathrm{e}^{\mathrm{N}(\mathrm{r}+\mathrm{s})}\left[1+\int_{-\infty}^{\mathrm{r}} \mathrm{G}_{2}(\mathrm{t}, \mathrm{s}) \mathrm{dt}\right]$

So the right hand side will be small if the integral in the brackets can be shown to be not very large. This can be seen by using (7) for $\mathrm{G}(\mathrm{r}, \mathrm{s})$ in the integral and evaluating the integral. This yields:

$\int_{-\infty}^{r} G_{2}(t, s) d t=\sum_{n=1}^{\infty} \frac{b_{n}}{n} e^{n(r+s)}$

The left hand side clearly converges faster than the series in (7) so it does not represent a large addition to $\mathrm{G}(\mathrm{r}, \mathrm{s})$ and so the left hand side of (12) can be taken as small.

\section{Conclusion}

In inverse scattering a real-world concern could be that small difference in the measured reflection coefficient could lead to very different predictions as to the potential. This brief paper has shown that this is not a concern. Despite the nonlinear nature of the equations, a small difference in reflection coefficients does correspond to a small difference in potentials.

An objection raised here may be that (6) and (7) are too restrictive in terms of possible spectral measure functions. It has been shown [6] that assuming this form of $\mathrm{G}(\mathrm{r}, \mathrm{s})$ can be a useful and effective way of calculation potentials for general cases.

\section{References}

[1] I.M. Gelfand, B.M. Levitan, on the determination of a differential equation by its spectral function, Dokl. Akad. Nauk. USSR 77 (1951) 557-560

[2] I.M. Gelfand, B.M. Levitan, on the determination of a differential equation by its spectral measure function, Izv. Akad. Nauk. SSR 15 (1951) 309-360.

[3] K. Chadan, P.C. Sabatier, Inverse Problems in Quantum Scattering Theory, Springer-Verlag, New York, 1977. http://dx.doi.org/10.1007/978-3-662-12125-2.

[4] R. Jost, W. Kohn, on the relation between phase shift energy levels and the potential, Danske Vid. Selsk. Math. Fys. 27 (1953) 3-19.

[5] E. Kincanon, an Orthogonal Set Composed from the Functionsenx, Applied Mathematics and Computation, 41, (1991) 69-75. http://dx.doi.org/10.1016/0096-3003(91)90107-X.

[6] E. Kincanon, Approximate solution it the Gelfand-Levitan equation, Applied Mathematics and Computation, 53 (1993) 121-128. http://dx.doi.org/10.1016/0096-3003(93)90097-X. 